\title{
Os Efeitos da Meditação à Luz da Investigação Científica em Psicologia: Revisão de Literatura
}

Meditation Effects on Scientific Research

in Psychology: Literature Review

Los Efectos de la Meditación a la Luz de la Investigación

Científica en Psicología: Revisión de Literatura

Carolina Baptista Menezes \&

Débora Dalbosco Dell'Aglio

Universidade Federal do Rio Grande do Sul
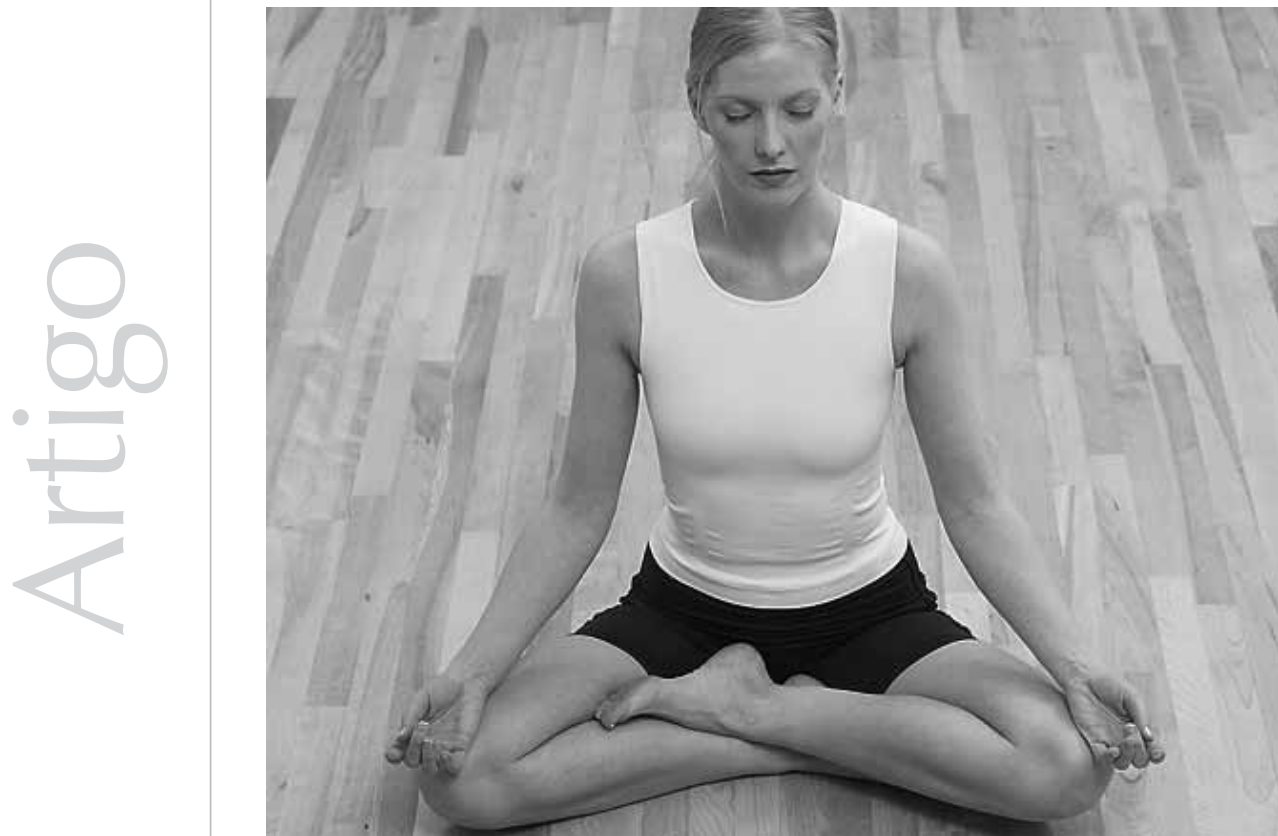
Resumo: A meditação, descrita como uma prática de auto-regulação do corpo e da mente, caracteriza-se por um conjunto de técnicas que treinam a focalização da atenção. Também conhecida como treinamento mental, essa prática constitui uma técnica capaz de produzir efeitos psicossomáticos. Este artigo apresenta a revisão de algumas evidências acerca dos benefícios da meditação e do seu papel na aplicação clínica. Inúmeros estudos vêm mostrando a sua eficácia, que está especialmente relacionada à diminuição dos sintomas ligados ao estresse e à ansiedade. Além disso, as pesquisas revelam que essa prática pode produzir efeitos de curta e longa duração que podem afetar positivamente as funções cognitivas e afetivas. Discutemse aspectos relacionados às definições e às particularidades de cada técnica e aos contextos aos quais pode estar atrelada. Apesar do crescente acúmulo de evidências sobre a relação entre meditação e saúde física e mental, ainda são necessárias mais investigações.

Palavras-chave: Meditação. Regulação da atenção. Saúde física. Saúde mental.

Abstract: Meditation, described as a practice of body and mind self-regulation, is characterized by a set of techniques that train the focalization of attention. Also known as a mental training, this practice characterizes a technique capable of producing psychosomatic effects. This paper presents a revision of some evidence on the benefits of meditation and its role for clinical application. A great deal of research has shown its efficacy, especially concerning its association with the reduction of symptoms related to stress and anxiety. Besides that, research has indicated that this practice can produce short and long term effects, affecting positively the cognitive and affective functions. We discuss the aspects related to the definitions and particularities of each technique and the contexts to which meditation can be linked to. Despite growing evidence on the relationship between meditation and physical and mental health, it is still necessary to carry out further investigation.

Keywords: Meditation. Attention regulation. Physical health. Mental health.

Resumen: La meditación, descrita como una práctica de autorregulación del cuerpo y de la mente, se caracteriza por un conjunto de técnicas que entrenan la focalización de la atención. También conocida como entrenamiento mental, esa práctica constituye una técnica capaz de producir efectos psicosomáticos. Este artículo presenta la revisión de algunas evidencias acerca de los beneficios de la meditación y de su papel en la aplicación clínica. Numerosos estudios vienen mostrando su eficacia, que está especialmente relacionada a la disminución de los síntomas relacionados al estrés y a la ansiedad. Además, las pesquisas revelan que esa práctica puede producir efectos de corta y larga duración que pueden afectar positivamente las funciones cognoscitivas y afectivas. Se discuten aspectos relacionados a las definiciones y a las particularidades de cada técnica y a los contextos a los cuales puede estar unida. A pesar de la creciente acumulación de evidencias sobre la relación entre meditación y salud física y mental, aún son necesarias más averiguaciones.

Palabras clave: Meditación. Regulación de la atención. Salud física. Salud mental.

Pode-se definir meditação como uma prática que engloba um conjunto de técnicas que buscam treinar a focalização da atenção (Shapiro, 1981). Por essa razão, pode ser chamada de processo auto-regulatório da atenção, em que através da prática é desenvolvido o controle dos processos atencionais (Davidson \& Goleman, 1977; Goleman \& Schwartz, 1976). Além disso, a meditação pode ser caracterizada como uma prática que atinge objetivos semelhantes a algumas técnicas da psicoterapia cognitiva, embora por meios distintos. Ambas levam à diminuição do pensamento repetitivo e à reorientação cognitiva, desenvolvendo habilidades para lidar com os pensamentos automáticos. A diferença, contudo, é que, na prática da meditação, os conteúdos que emergem à consciência não devem ser confrontados ou elaborados intencionalmente, apenas observados, de forma que a prática se transforme em um aprendizado de como não deixar influenciar-se pelos mesmos e compreendê-los como fluxos mentais (Bishop et al., 2004; Miller, Fletcher, \& Kabat-Zinn, 1995; Vandenbergue \& Sousa, 2006).

Também designada como técnica mentecorpo (Hankey, 2006; Kabat-Zinn, 2003), técnica comportamental (Cardoso, 2005; Vandenbergue \& Sousa, 2006) e resposta de relaxamento (Campagne, 2004; Galvin, Benson, Deckro, Fricchione, \& Dusek, 2006), a meditação é descrita como um treinamento 
mental, capaz de produzir maior integração entre mente, corpo e mundo externo (Brefczynski-Lewis, Lutz, Schaeffer, Levinson, \& Davidson, 2007; Slagter et al., 2007).

A meditação é uma prática muito antiga, com origem nas tradições orientais, estando especialmente relacionada às filosofias do yoga e do budismo (Levine, 2000). Contudo, esse termo também é utilizado para designar algumas práticas cultivadas por certas religiões, como o cristianismo, o judaísmo, o islamismo, o taoísmo e o xamanismo, entre outras, através do deslocamento da consciência do mundo externo para o interno (Naranjo, 2005). Com relação às investigações científicas, embora conste que desde 1936 o potencial da meditação vem sendo discutido (Smith, 1975), apenas por volta da década de 60 a meditação começou a ser objeto de estudos mais rigorosos (Goleman, 1988; Shapiro, 1981). Fazendo uma busca sobre o descritor "meditação" na base de dados PsycINFO, verificou-se que, dos 1588 resumos listados em peer reviewed journals, o mais antigo data de 1956. Além disso, a sua concepção apresenta ênfases diferentes. Enquanto no Oriente meditar é sinônimo de busca espiritual, no Ocidente, em especial nas pesquisas científicas, a palavra meditação tem sido utilizada para descrever práticas auto-regulatórias do corpo e da mente. A investigação científica da meditação parte da premissa que, embora existam diversas técnicas, todas têm uma característica fundamental comum: o controle da atenção (Cahn \& Polich, 2006; Goleman, 1988).

Conforme o processo atencional é direcionado, as técnicas meditativas podem ser classificadas em dois ou três tipos principais, na visão ocidental. Em geral, dois estilos básicos são mencionados: mindfulness e concentrativo (Cahn \& Polich, 2006; Davidson \& Goleman, 1977). O tipo mindfulness, ou meditação do insight, é descrito como uma prática de abertura, em que há uma percepção dos estímulos, como pensamentos, sentimentos e/ou sensações, embora a atenção específica mantida seja uma observação livre que não os julga nem analisa. Algumas técnicas orientais que se enquadram nesse tipo são a meditação zen, vipassana e a própria adaptação ocidental mindfulness. As técnicas meditativas concentrativas caracterizam-se pela restrição da atenção a um único objeto, interno ou externo. Ignora-se qualquer estímulo do ambiente, focalizando uma atividade mental ou sensorial específica, por exemplo, a repetição de um som, uma imagem ou a respiração. Nesse tipo, incluem-se algumas meditações oriundas do yoga, como a meditação transcendental e a meditação budista samatha (Cahn \& Polich, 2006). Já alguns autores argumentam que existe um terceiro tipo, denominado contemplativo, que seria uma integração dos dois outros tipos, visto que requer tanto a habilidade de focalizar como de se abrir. Alguns exemplos são a meditação judaica e determinadas orações (Shapiro, 1981; Shapiro, Schwartz, \& Santerre, 2005).

Na prática, esses tipos básicos podem interagir, constituindo facetas de um único processo (Goleman, 1988) ou de um contínuo, ao longo do qual se encontram diversas técnicas com seus subtipos (Cahn \& Polich, 2006; Naranjo, 2005). Muitas vezes é preciso treinar a concentração primeiramente, para só então conseguir abrir-se à observação livre de julgamento dos conteúdos mentais, como propõe o tipo mindfulness (Goleman, 1988). Na investigação científica, os estudos têm pesquisado os efeitos dos diferentes tipos isoladamente. Acredita-se que cada técnica possua determinadas especificidades que repercutem no desenvolvimento de habilidades particulares (Hankey, 2006; Newberg et al., 2001). Bishop et al. (2004), por exemplo, propuseram uma definição operacional específica para a meditação do tipo mindfulness, que pode ser compreendida e medida através de dois componentes 
fundamentais: a auto-regulação da atenção e a atitude de abertura e aceitação do momento presente.

Além das especificidades de cada técnica, outras variáveis, como predisposições genéticas, traços de personalidade, vivências particulares de cada um, expectativas, motivações e valores também podem mediar o tipo e a qualidade da resposta que a prática meditativa produzirá (Davidson \& Goleman, 1977; Smith, 1978; Takahashi et al., 2005). Portanto, o estudo da meditação e de seus efeitos deve considerar as características do sujeito antes da prática, os estados que ocorrem durante ou pouco tempo após a meditação, e, por fim, as mudanças duradouras resultantes da prática continuada (Davidson \& Goleman, 1977). É consenso que a melhor forma de fazê-lo é através de estudos longitudinais (Brefczynski-Lewis et al., 2007; Davidson \& Goleman, 1977; Hankey, 2006; Lazar et al., 2005; Slagter et al., 2007).

A partir da revisão de literatura, observase que muitos resultados encontrados a partir de diferentes técnicas são bastante semelhantes. Com base nessa percepção e no fato de que técnicas distintas possuem características essenciais comuns (Goleman, 1988), alguns pesquisadores brasileiros concluíram que seria importante criar uma definição operacional geral acerca da meditação que abarcasse tais características e que permitisse uma padronização do seu uso para fins de pesquisa em saúde (Cardoso, Souza, Camano, \& Leite, 2004). Assim, igualmente sob a prerrogativa da auto-regulação, descrevem a prática de meditação como a utilização de alguma técnica específica e claramente definida, com a qual se alcança algum relaxamento muscular e mental durante o processo, sendo um estado exclusivamente auto-induzido através da utilização de um foco (âncora).
Embora essa operacionalização seja um importante passo no sentido de sistematizar e padronizar o estudo da meditação, certamente o seu campo de investigação ainda está em desenvolvimento. Portanto, ainda são necessárias pesquisas que esclareçam alguns pontos que permanecem sem consenso por parte da comunidade científica, como as especificidades de cada técnica e seus respectivos efeitos, o grau com que se diferenciam e o impacto que tal diferença pode ter no resultado final da prática de longa duração. Entretanto, até o momento presente, o que se percebe é um grande corpo de pesquisas que, mesmo não intencionalmente, sugere alguma uniformidade entre os efeitos das práticas meditativas. Dessa forma, parece correto concluir, assim como propõem Cardoso et al. (2004), que existem alguns fatores comuns e essenciais nas diferentes técnicas meditativas que as tornam igualmente eficazes e que, de alguma forma, caracterizam o que é meditação.

\section{Correlatos neurofisiológicos da meditação}

Os primeiros estudos sobre meditação estiveram voltados para explicar os mecanismos subjacentes a essa prática e suas repercussões na vida do praticante. Através de medidas minuciosas e rigorosas, foi identificada uma série de padrões de reações associados à prática meditativa, que a caracterizam como um estado de consciência particular, diferente dos tradicionalmente conhecidos, como vigília, sono e sonho (Wallace, 1970). Essas reações também são chamadas de respostas psicofisiológicas ou neurofisiológicas, pois refletem mudanças no sistema nervoso central e autônomo (Aftanas \& Golocheikine, 2001; Danucalov \& Simões, 2006).

As alterações do sistema nervoso autônomo, constatadas por estudos experimentais e de 
meta-análise, incluem redução do consumo de oxigênio, da eliminação de gás carbônico e da taxa respiratória, o que indica uma diminuição da taxa do metabolismo. Além disso, a meditação também está associada a um aumento da resistência da pele e a uma redução do lactato plasmático, cuja alta concentração é associada a altos níveis de ansiedade (Dillbeck \& Orme-Johnson, 1987; Travis \& Wallace, 1999; Wallace, 1970; Wallace \& Benson, 1972; Wallace, Benson, \& Wilson, 1971). Com respeito à freqüência cardíaca, alguns estudos não observaram diferença (Dillbeck \& OrmeJohnson, 1987; Travis \& Wallace, 1999), enquanto outros verificaram uma diminuição significativa (Maura et al., 2006; Wallace, 1970; Wallace \& Benson, 1972; Wallace et al., 1971). Embora já tenha sido discutido que os resultados sobre os efeitos da meditação não são conclusivos (Canter, 2003; Holmes, 1984), na literatura, há um crescente número de pesquisas corroborando os correlatos fisiológicos dessa prática (MacLean et al., 1997; Goleman \& Schwartz, 1976; Takahashi et al., 2005; Travis \& Wallace, 1999).

Em geral, a observação dessas reações levou à conclusão de que através da meditação é possível atingir um estado de hipometabolismo basal ao mesmo tempo em que a mente se mantém alerta e que aquele que medita desenvolve, portanto, a capacidade de controlar determinadas funções fisiológicas involuntárias (Wallace et al., 1971). É com base nessa idéia, ou seja, na capacidade de obter algum grau de controle sobre processos psicobiológicos autonômicos, que a meditação pode ser considerada uma técnica eficaz de biofeedback, constituindo uma das técnicas mais antigas de autoregulação (Cahn \& Polich, 2006; Davidson \& Goleman, 1977).

Além das mudanças autonômicas, desde a década de 70, também ganhou destaque a investigação dos efeitos cerebrais da meditação, sob a premissa de que estados mentais podem alterar as funções fisiológicas (Wallace et al., 1971). Foi verificado, então, que algumas características tradicionalmente associadas à meditação, como baixa ansiedade e afetos positivos, poderiam ser explicadas por mudanças da atividade neuroelétrica. Através do Exame de Eletroencefalograma (EEG), o aumento da produção de ondas alfa nas regiões frontais e, em menor quantidade, de ondas teta, foi observado tanto em iniciantes quanto avançados (Aftanas \& Colocheikine, 2001; Hankey, 2006; Takahashi et al., 2005; Wallace \& Benson, 1972), sendo a observação de ondas teta mais comum em meditadores com maior experiência (Cahn \& Polich, 2006).

Atualmente, sabe-se que, além dessas mudanças funcionais, a meditação também pode produzir mudanças estruturais, atuando sobre a plasticidade cerebral. Uma pesquisa que comparou a espessura do córtex de meditadores experientes com um grupocontrole encontrou uma diferença significativa nas regiões relacionadas à sustentação da atenção, onde a espessura era maior nos praticantes experientes (Lazar et al., 2005). Esse estudo corrobora a idéia de que a regularidade e a continuidade da prática influenciam a intensidade das respostas e que, portanto, a meditação pode produzir mudanças duradouras. Várias pesquisas observaram que, mesmo em situações basais, os meditadores mais experientes produziam respostas significativamente diferentes daquelas medidas em controles (Cahn \& Polich, 2006; Dillbeck \& OrmeJohnson, 1987; Easterlin \& Cardeña, 1998; Goleman \& Schwartz, 1976; Lutz, Greischar, Rawlings, Ricard, \& Davidson, 2004).

A atenção é uma das funções cognitivas que parece estar particularmente envolvida nas mudanças que a prática meditativa pode gerar. Já na década de 70, foi demonstrado, através de alguns testes neuropsicológicos, que, quanto maior o tempo de prática 
de meditação, maior a capacidade de absorção atencional, estando esta associada à diminuição da ansiedade (Davidson, Goleman, \& Schwartz, 1976). Pesquisas mais recentes têm confirmado essa idéia através de medidas cognitivas e neurais. Por meio do Exame de Tomografia Computadorizada por Emissão de Fóton Único (SPECT) (Newberg et al., 2001) e por medição de ondas gama (Lutz et al., 2004), esses dois estudos verificaram que meditadores budistas experientes tinham respostas cerebrais que indicavam um poder significativamente maior de concentração, em comparação com o grupo-controle.

Dois estudos que investigaram praticantes da meditação concentrativa também observaram diferenças significativas na habilidade atencional (Brefczynski-Lewis et al., 2007; Carter et al., 2005). Além disso, o estudo de Brefczynski-Lewis et al. corroborou uma das idéias centrais da meditação concentrativa, a de que, quanto maior o tempo de prática, menor o esforço exigido para manter maior foco. Os autores verificaram que os praticantes com maior número de horas apresentaram menor ativação das regiões envolvidas no processo meditativo, sugerindo que a técnica pode agir sobre a distribuição de recursos cognitivos, cuja idéia já havia sido demonstrada em outro estudo que utilizava a técnica mindfulness.

Através da tarefa do "piscar atencional", foi constatado que as pessoas que participaram de um treino intensivo de três meses de meditação mindfulness tiveram uma redução da alocação dos recursos do cérebro para o primeiro estímulo da tarefa, com um conseqüente aumento da capacidade de detectar o segundo estímulo apresentado, sem que houvesse nenhum comprometimento na habilidade de perceber o primeiro (Slagter et al., 2007). Os autores concluíram que a habilidade de processar dois estímulos significativos e muito próximos temporalmente depende da eficiência com que os recursos mentais são empregados na percepção do primeiro estímulo, sendo a meditação uma forma de aumentar o controle sobre a distribuição desses recursos.

Embora com técnicas diferentes, essas pesquisas mostram resultados complementares, indicando que através da meditação é possível treinar habilidades cognitivas, como a atenção. Outros estudos se preocuparam em comparar as diferenças nos padrões cognitivos e na atividade neural entre os distintos tipos de meditação. Carter et al. (2005) verificaram que metade dos meditadores que praticaram o tipo concentrativo conseguiu manter uma estabilidade perceptiva significativamente prolongada no teste utilizado, comparado aos praticantes da meditação budista da compaixão (Carter et al., 2005). Outro estudo, ao comparar quatro subtipos da meditação budista, constatou que a atividade da freqüência gama (25-42 Hz) não apresentava uma distribuição igual entre as diferentes técnicas (Lehmann et al., 2001).

A partir desses resultados, pode-se inferir que existem algumas distinções nos mecanismos subjacentes a diferentes técnicas, mesmo que os resultados ainda não sejam conclusivos. Entretanto, também se pode notar que técnicas distintas parecem ser capazes de atuar na atenção, corroborando a idéia de que, mesmo havendo especificidades em cada prática, todas possuem a auto-regulação da atenção como processo básico comum.

\section{Meditação, saúde física e saúde mental}

Sintomas de estresse, em particular, têm apresentado resultados bastante significativos após o uso da meditação com populações clínicas e não clínicas, como apontam as medidas de sofrimento (distress) psicológico e marcadores biológicos (Cruess, Antoni, Kumar, \& Schneiderman, 2000; Goleman 
\& Schwartz, 1976; Oman, Hedberg, \& Thoresen, 2006; Ostafin et al., 2006). Além disso, segundo uma meta-análise em que foi observado um alto e consistente tamanho de efeito da meditação sobre diversas situações clínicas, é através da redução do estresse que a meditação pode ser benéfica para diversas condições de saúde (Grossman, Niemannb, Schmidtc, \& Walachc, 2004).

Estudos de acompanhamento descobriram que a meditação auxilia no gerenciamento e na redução do estresse, e que esse efeito se prolonga no tempo (Miller et al., 1995; Oman et al., 2006; Ostafin et al., 2006). Embora Ostafin et al. (2006) não tenham encontrado uma correlação positiva significativa entre a freqüência da prática e a diminuição do sofrimento psicológico, Oman et al. (2006) constataram que a adesão à prática de meditação ao longo de quatro meses teve efeito direto na redução do estresse após esse tempo.

Outras pesquisas indicaram que a meditação pode proporcionar melhor adaptação ao estresse. Através de um estímulo aversivo, foi verificado que o grupo com maior experiência se recuperava mais rápido da excitação autonômica, ou seja, os meditadores experientes, após o término do estressor, tinham a freqüência cardíaca e a resposta de condutividade da pele diminuída mais rapidamente, o que indicava uma capacidade de habituação mais rápida ao estresse (Goleman \& Schwartz, 1976). Nessa mesma direção, um estudo verificou que, mesmo quando praticantes iniciantes e avançados relatavam menor aceitação em condições de estresse, o grupo menos experiente apresentava significativamente menos aceitação (Easterlin \& Cardeña, 1998).

Esses resultados são corroborados por estudos que mediram a relação entre meditação e cortisol e que observaram uma diminuição desse hormônio em pacientes HIV (Cruess et al., 2000) com câncer de próstata e mama (Carlson, Speca, Patel, \& Goodey, 2004) e praticantes não clínicos (MacLean et al., 1997) em comparação aos controles. Ademais, essa redução esteve sempre associada à diminuição dos sintomas de sofrimento psicológico. Também foi constatado que a variância diurna da secreção de adrenocorticotrópico (ACTH) e ß-endorfina foi significativamente menor em meditadores em comparação a um grupocontrole, e que, portanto, essa técnica pode ter um efeito neuroendócrino modulador do eixo hipotálamo-hipófise-adrenal (Infante et al., 1998).

A prática meditativa também está associada à diminuição da ansiedade (Brown \& Ryan, 2003; Davidson et al., 2003; Galvin et al., 2006; Schwartz, Davidson, \& Goleman, 1978; Grossman et al., 2004), sendo que os efeitos de uma intervenção de oito semanas de meditação sobre a redução dos sintomas do transtorno de ansiedade generalizada e do transtorno de pânico, com e sem agorafobia, foram mantidos por três anos (Miller et al., 1995). Além disso, pessoas com o transtorno do comer compulsivo que passaram por uma intervenção que utilizava meditação tiveram a freqüência e a intensidade de seus episódios diminuídas em função da redução da ansiedade e da depressão (Baer, Fischer \& Huss, 2005; Kristeller \& Hallett, 1999).

A meditação também é capaz de estimular aspectos saudáveis, estando muito associada à saúde mental (Goleman, 1988; Hankey, 2006). Uma pesquisa verificou que a prática meditativa associou-se à ativação do córtex pré-frontal esquerdo (Davidson et al., 2003), que está relacionado a afetos positivos e a maior resiliência (Davidson, 2004). Além disso, ondas teta produzidas pela meditação também mostraram correlação positiva com o relato da experiência emocional positiva durante a prática de meditadores experientes, em contraste com iniciantes (Aftanas \& Golocheikine, 2001). 
Segundo Jain et al. (2007), a meditação pode proporcionar o desenvolvimento de características psicológicas positivas por meio da redução de pensamentos ruminativos e de distração.
Medidas subjetivas e com instrumentos de auto-relato também indicaram que a meditação é capaz de gerar afetos positivos (Brazier, Mulkins, \& Verhoef, 2006; Easterlin \& Cardeña, 1998; Jain et al., 2007), melhorar o humor (Cruess et al., 2000), melhorar a qualidade de vida (Carlson et al., 2004) e o bem-estar psicológico (Brazier et al., 2006; Wallace, 1970), sendo que, quanto maior o tempo de prática, maior o relato da experiência emocional positiva (Aftanas \& Golocheikine, 2001; Brown \& Ryan, 2003). Além disso, uma pesquisa que comparou o afeto positivo entre meditadores experientes e um grupo-controle revelou que, mesmo em níveis basais, os meditadores apresentavam níveis significativamente maiores de afeto positivo (Goleman \& Schwartz, 1976), corroborando o pressuposto das filosofias orientais de que a meditação pode trazer efeitos que se sobrepõem à condição de estado.

Assim, através da prática continuada, é possível transformar estados em traços, que atuam, inclusive, sobre a personalidade. Em comparação a um grupo-controle, meditadores experientes apresentaram menor neuroticisimo (Goleman \& Schwartz, 1976; Leung \& Singhal, 2004), sendo que, quanto maior o tempo de prática, menor era a prevalência desse traço (Leung \& Singhal, 2004). Outro estudo verificou que meditadores mais experientes se mostraram mais adaptados, alegres, maduros, autoconfiantes e com melhor auto-imagem. Também foi observado que esses praticantes, em comparação com o grupo controle, eram mais estáveis emocionalmente, conscientes, confiantes, relaxados e auto-suficientes (Sridevi \& Krisha Rao, 1998).

Segundo Jain et al. (2007), a meditação pode proporcionar o desenvolvimento de características psicológicas positivas por meio da redução de pensamentos ruminativos e de distração. Ao comparar os efeitos da meditação e do relaxamento corporal, Jain et al. verificaram que, embora ambas as técnicas tenham produzido uma redução do sofrimento psicológico e um aumento de afetos positivos, a meditação teve um efeito maior no aumento dos afetos positivos. Além disso, foi a única técnica com efeito sobre a redução de pensamentos e comportamentos ruminativos.

Para Bishop et al. (2004), a consciência não-ruminativa desenvolve-se através de um processo meta-cognitivo característico da meditação, uma vez que esta trabalha fundamentalmente a auto-observação dos processos mentais. Essa idéia é consistente com os resultados que mostram um aumento da autoconsciência associada à prática meditativa (Brown \& Ryan, 2003; Easterlin \& Cardeña, 1998). Além disso, confirmam o pressuposto que, através dessa atividade, é possível atingir importantes níveis de insight (Goleman, 1988).

Em razão dessa relação entre meditação e aspectos psicológicos positivos, muitos autores a concebem como uma técnica útil para tratamentos psicoterápicos (Hayward \& Varela, 2001; Martin, 1997; Naranjo, 2005). A meditação, assim como a psicoterapia, busca a eliminação das barreiras do ego, a fim de que as potencialidades humanas se manifestem (Martin, 1997; Naranjo, 2005). Através da focalização da atenção, a meditação pode ser interpretada como uma tentativa de desfazer os condicionamentos e as programações da mente (Goleman, 2003); além disso, o desenvolvimento de uma atenção livre de elaboração pode possibilitar o surgimento de conteúdos antes inacessíveis à consciência (Bishop et al., 2004); portanto, alguns autores acreditam que a meditação se aproxima dos pressupostos norteadores de diversas linhas teóricas da Psicologia (Naranjo, 2005; Vandenberghe \& Sousa, 2006), podendo ser descrita como um estado de liberdade psicológica (Martin, 1997). 


\section{Aplicações}

Além da relação com a psicoterapia, a meditação também passou a ser uma intervenção clínica utilizada como ferramenta para tratamentos coadjuvantes, tornando-se o foco principal de determinados programas de saúde. O Mindfulness-Based Stress Reduction (MBSR - Programa de Redução de Stress Baseado na mindfulness), por exemplo, é um programa desenvolvido por Kabat-Zinn, situado em uma clínica de redução de estresse para pacientes não hospitalizados no Centro Médico da Universidade de Massachusetts, cujo objetivo é proporcionar alívio ao sofrimento físico e psicológico (Kabat-Zinn, 2003). O programa é constituído de oito encontros semanais, de duas horas e meia, nas quais são treinadas as técnicas de posturas de yoga e meditação que devem ser praticadas no restante dos dias durante as oito semanas, por uma hora, em casa. Além disso, um retiro silencioso de sete horas é realizado na sexta semana. Inúmeros desfechos positivos de saúde, como a melhora da psoríase (Kabat-Zinn et al., 1998), dos transtornos de ansiedade (Miller, et al., 1995) e redução do estresse, tanto em pacientes não clínicos (Grossman et al., 2004; Jain et al., 2007) quanto clínicos (Carlson et al., 2004; Speca, Carlson, Goodey, \& Angen, 2000), têm sido associados ao programa, o que sugere sua eficácia.

OMBSR também deu origem a outro programa similar, porém voltado para o tratamento da depressão, dentro de um contexto ligado à psicoterapia cognitivo-comportamental. Esse programa, intitulado Mindfulness-Based Cognitive Therapy (MBCT - Terapia Cognitiva Baseada na Mindfulness), tem como objetivo fazer um acompanhamento de pacientes depressivos que tiveram êxito na psicoterapia cognitiva, a fim de prevenir a sua recaída. Assim como o MBSR, o MBCT inclui oito encontros semanais, embora com grupos mais reduzidos, sendo que o participante não pode estar deprimido à época do treinamento. Esse programa diferencia-se da terapia cognitivo-comportamental na medida em que não enfatiza a mudança do conteúdo dos pensamentos, e sim, a transformação da consciência que o paciente possui acerca dos pensamentos e a forma como se relaciona com estes. O programa tem se mostrado eficaz para pacientes que tiveram pelo menos três episódios de depressão através do aumento do acesso à meta-cognição, em que pacientes vivenciam pensamentos e sentimentos negativos como meros estados mentais, e não como uma condição imutável do self (Teasdale et al., 2002).

Outro programa bastante conhecido em Harvard, no centro médico Mind Body Medical Institute, foi desenvolvido no final da década de 60 pelo médico cardiologista Herbert Benson, e chama-se resposta de relaxamento (Casey \& Benson, 2004). Benson cunhou o termo "resposta de relaxamento" para designar o conjunto de técnicas utilizadas, como a meditação e o relaxamento progressivo, no tratamento coadjuvante de diversas condições, buscando proporcionar maior saúde e bem-estar aos pacientes. $\mathrm{O}$ autor descreve a resposta de relaxamento como um conjunto de reações biológicas e emocionais opostas àquelas geradas pela resposta de luta ou fuga característica do estresse. Uma série de aplicações clínicas do programa tem mostrado resultados significativos, como menor somatização (Nakao et al., 2001), melhor manejo da dor crônica e aguda (Schaffer \& Yucha, 2004), melhora de condições relacionadas ao estresse (Esch, Fricchione, \& Stefano, 2003), entre outros.

A meditação e outras técnicas orientais e de relaxamento também vêm sendo utilizadas em sistemas de saúde de alguns países, como Nova Zelândia (Duke, 2005), Canadá (Andrewsa \& Boonb, 2005), Austrália (McCabe, 2005), Reino Unido (Ernst, Schmidt, 
Um estudo revelou que as pessoas que utilizaram a meditação de cunho espiritual tiveram maior redução da ansiedade, maior relato de afetos positivos e maior tolerância à dor, em comparação com o grupo que utilizou a meditação secular

(Wachholtz \& Pargament, 2005).
\& Wider, 2005) e Estados Unidos (Spiegel, Stroud, \& Fyfe, 1998), sob a designação de medicina complementar alternativa (CAM). No Brasil, o Ministério da Saúde, através da Portaria no 971, com base em um documento da Organização Mundial da Saúde, aprova a utilização de práticas complementares da Medicina chinesa, como acupuntura, homeopatia e meditação (Ministério da Saúde, 2006). Todavia, ainda não há publicações científicas que retratem a utilização da meditação no sistema de saúde, embora certamente seja utilizada na prática clínica pública e privada. Nas universidades brasileiras, também há escassez de investigações acerca do assunto. Tem-se notícia de dois grupos de pesquisa que vêm trabalhando na área, um bastante voltado para a pesquisa, no Departamento de Psicobiologia da Universidade Federal de São Paulo (UNIFESP), e outro mais direcionado para a prática e a inserção da técnica no âmbito da saúde mental, na Clínica-Escola de Psicologia da Universidade de Fortaleza (UNIFOR), onde é oferecido um serviço terapêutico gratuito à população.

\section{Conclusões}

Sugere-se que a meditação pode ser entendida e vivenciada a partir de duas perspectivas, que podem se complementar. A prática pode ser o reflexo de um contexto religioso-espiritual, através da qual são cultivados os ensinamentos transmitidos pelos preceitos filosóficos característicos da tradição e/ou a meditação pode ser uma atividade inserida no âmbito da saúde, na condição de técnica capaz de produzir determinados benefícios, promovendo maior saúde física e mental. Parece prudente inferir que uma não necessita excluir a outra, e tampouco uma necessariamente precisa da outra.

Embora a meditação seja oriunda de filosofias orientais, que caracterizam uma forma particular de perceber e de portar-se no mundo, incluindo valores éticos, os resultados aqui discutidos refletem outro aspecto da prática, indicando seu potencial como uma técnica psicossomática com repercussões importantes para o campo da saúde em geral. A técnica em si é capaz de gerar uma série de respostas físicas e psicológicas que podem auxiliar na prevenção de inúmeras condições, especialmente aquelas resultantes dos efeitos deletérios do estresse, no manejo de problemas de saúde já estabelecidos, assim como na promoção de saúde mental. Dessa forma, a meditação é uma atividade que pode ser utilizada em um contexto terapêutico, desde que as condições e as peculiaridades da situação que está sendo tratada sejam respeitadas.

Um estudo revelou que as pessoas que utilizaram a meditação de cunho espiritual tiveram maior redução da ansiedade, maior relato de afetos positivos e maior tolerância à dor, em comparação com o grupo que utilizou a meditação secular (Wachholtz \& Pargament, 2005). Além disso, alguns autores sustentam que a meditação realizada fora de seu contexto original não é tão facilitadora de características tradicionalmente atreladas à prática, como níveis mais profundos de propósito na vida e auto-atualização (Shapiro et al., 2005). Entretanto, propõe-se que a meditação seja concebida como um processo multifatorial, em que muitos aspectos se inter-relacionam, mediando seus efeitos, como genética e traços de personalidade, motivações e valores, tanto pré-existentes como adquiridos com a própria prática e com as experiências individuais, além do tipo da técnica. Embora a espiritualidade possa ter um impacto positivo sobre o bem-estar e a saúde física e mental (Guimarães \& Avezum, 2007; Peres, Simão, \& Nasello, 2007), existe um limite que se refere às pessoas que não querem levar uma vida calcada na espiritualidade e que nem por isso devem ser impedidas de meditar ou de ter acesso à 
prática meditativa. Ademais, são necessários mais estudos comparando os diferentes tipos quanto aos seus mecanismos subjacentes e quanto a uma possível distinção do impacto de cada um.

Além das especificidades de cada tipo, ressalta-se que os estudos sobre meditação ainda suscitam opiniões divergentes quanto à aceitação da sua aplicabilidade e eficácia no campo da saúde. Alguns autores advertem que ainda são necessárias pesquisas com metodologias mais rigorosas para assegurar que a meditação seja, de fato, a responsável pelos efeitos observados (Baer, 2003; Bishop, 2002; Canter \& Ernst, 2004). Entre os principais argumentos, estão a nãorandomização de muitas amostras, o número reduzido de participantes, a afiliação dos pesquisadores às organizações ligadas à técnica investigada e, até 2004, argumentavase com a falta de uma definição operacional objetiva (Bishop et al., 2004; Cardoso et al., 2004).

Por outro lado, uma grande quantidade de estudos com resultados positivos sugere que a meditação pode ser eficiente no que tange a reações psicossomáticas e, especialmente, a experiências subjetivas, como a sensação de bem-estar e de crescimento pessoal. Com relação ao tempo necessário para que tais efeitos ocorram, não há uma precisão. Embora pesquisas revelem que com alguns meses já se pode observar uma diferença significativa em determinados estados, também há estudos indicando que, quanto maior o tempo de prática, maior a intensidade e a permanência das respostas produzidas. Portanto, a regularidade da prática constituise em mais uma variável mediadora dos efeitos da meditação e, possivelmente, em um diferencial na medida em que tais reações se transformam em aspectos mais duradouros e estáveis da personalidade.

Tendo em vista a relevância do estudo científico da meditação e de suas diversas facetas, sugere-se que esse é um campo a ser explorado nos centros de pesquisa do Brasil. O estágio ainda em desenvolvimento em que se encontram os estudos sobre meditação gera um solo fértil para a sua investigação, e possibilita, à medida que os resultados vão se tornando mais conclusivos, a sua inserção e disseminação na prática clínica. Além disso, recomenda-se que aqueles profissionais que já a utilizam em suas práticas terapêuticas relatem seus casos com o rigor científico necessário para que se inicie um movimento brasileiro de validação do uso da meditação como uma ferramenta destinada a promover a qualidade de vida nas suas diversas dimensões. 


\section{Carolina Baptista Menezes}

Mestre em Psicologia pela UFRGS, doutoranda em Psicologia com bolsa CNPq pela UFRGS

E-mail: menezescarolina@hotmail.com

\section{Débora Dalbosco Dell'Aglio}

Professora do Programa de Pós-Graduação do Instituto de Psicologia, Universidade Federal do Rio Grande do Sul, em Psicologia, coordenadora do Núcleo de Estudos e Pesquisas em Adolescentes (NEPA)

E-mail: dalbosco@cpovo.net

\section{Endereço para envio de correspondência:}

Instituto de Psicologia da Universidade Federal do Rio Grande do Sul

Ramiro Barcelos, 2600, sala 115

Bairro Santa Cecília

Porto Alegre, RS - Brasil, CEP: 90035-003

Recebido 22/04/2008 Reformulado 26/12/2008 Aprovado 06/01/2009

Aftanas, L. I., \& Golocheikine, S. A. (2001). Human anterior and frontal midline theta and lower alpha reflect emotionally positive state and internalized attention: High-resolution EEG investigation of meditation. Neuroscience Letters, 310, 57-60.

Andrewsa, G. J., \& Boonb, H. (2005). CAM in Canada: Places, practices, research. Complementary Therapies in Clinical Practice, 11, 21-27.

Baer, R. A. (2003). Mindfulness training as a clinical intervention: A conceptual and empirical review. Clinical Psychology: Science and Practice, 10, 125-143.

Baer, R. A., Fischer, S., \& Huss, D. B. (2005). Mindfulness-based cognitive therapy applied to binge eating: a case study. Cognitive and Behavioral Practice, 12, 351-358.

Bishop, S. R. (2002). What do we really know about mindfulnessbased stress reduction? Psychosomatic Medicine, 64, 71-84.

Bishop, S. R., Lau, M., Shapiro, S., Carlson, L., Anderson, N. D., Carmody, J. et al. (2004). Mindfulness: A proposed operational definition. Clinical Psychology: Science and Practice, 11(3), 230-241.

Brazier, A., Mulkins, A., \& Verhoef, M. (2006). Evaluating a yogic breathing and meditation intervention for individuals living with HIV/AIDS. American Journal of Health Promotion, 20(3), 192-195.
Brefczynski-Lewis, J. A., Lutz, A., Schaeffer, H. S., Levinson, D. B., \& Davidson, R. J. (2007). Neural correlates of attentional expertise in long-term meditation practitioners. Proceedings of the National Academy of Sciences, 104(27), 11483-11488.

Brown, K. W., \& Ryan, R. M. (2003). The benefits of being present: Mindfulness and its role in psychological well-being. Journal of Personality and Social Psychology, 84(4), 822-848.

Cahn, B. R., \& Polich, J. (2006). Meditation states and traits: EEG, ERP and neuroimaging studies. Psychological Bulletin, 132(2), 180-211.

Campagne, D. M. (2004). Teoría y fisiología de la meditación. Cuadernos de Medicina Psicossomatica y Psiquiatria de Enlace, (69/70), 15-30.

Canter, P. (2003). The therapeutic effects of meditation: The conditions treated are stress related, and the evidence is weak. British Medical Journal, 326, 1049-1050.

Canter, P. H., \& Ernst, E. Insufficient evidence to conclude whether or not transcendental meditation decreases blood pressure: Results of a systematic review of randomized clinical trials. Journal of Hypertension, 22, 2049-2054.

Cardoso, R. (2005). Medicina e meditação: um médico ensina a meditar (2a ed., 134 pp.). São Paulo: MG Editores. 
Cardoso, R., Souza, E., Camano, L., \& Leite, J. R. (2004). Meditation in health: An operational definition. Brain Research Protocols, 14, 58-60.

Carlson, L. E., Speca, M., Patel, K. D., \& Goodey, E. (2004). Mindfulness-based stress reduction in relation to quality of life, mood, symptoms of stress and levels of cortisol, dehydroepiandrosterone sulfate (DHEAS) and melatonin in breast and prostate cancer outpatients. Psychoneuroendocrinology, 29, 448-474.

Carter, O. L., Presti, D. E., Callistemon, C., Ungerer, Y., Liu, G. B., \& Pettigrew, J. D. (2005). Meditation alters perceptual rivalry in Tibetan buddhist monks. Current Biology, 15, R412-413.

Casey, A., \& Benson, H. (2004). Mind your heart. New York: Free Press.

Cruess, D. G., Antoni, M. H., Kumar, M., \& Schneiderman, N. (2000). Reductions in salivary cortisol are associated with mood improvement during relaxation training among HIV-seropositive men. Journal of Behavioral Medicine, 23(2), 107-122.

Danucalov, M. A. D., \& Simões, R. S. (2006). Neurofisiologia da meditação. São Paulo: Phorte.

Davidson, R. J. (2004). Well-being and affective style: Neural substrates and biobehavioural correlates. Philosophical Transactions of the Royal Society B, 359(1449), 1395-1411.

Davidson, R. J., \& Goleman, D. J. (1977). The role of attention in meditation and hypnosis: A psychobiological perspective on transformations of consciousness. The International Journal of Clinical and Experimental Hypnosis, 25(4), 291-308.

Davidson, R. J., Goleman, D. J., \& Schwartz, G. E. (1976). Attentional and affective concomitants of meditation: A cross-sectional study. Journal of Abnormal Psychology, 85(2), 235-238.

Davidson, R. J., Kabat-Zinn, J., Schumacher, J., Rosenkranz, M., Muller, D., Santorelli, S. F. et al. (2003). Alterations in brain and immune function produced by mindfulness meditation. Psychosomatic Medicine, 65, 564-570.

Dillbeck, M. C., \& Orme-Johnson, D. W. (1987). Physiological differences between transcendental meditation and rest. American Psychologist, 42(9), 879-881.

Duke, K. (2005). A century of CAM in New Zealand: A struggle for recognition. Complementary Therapies in Clinical Practice, 11, 11-16.

Easterlin, B. L., \& Cardeña, E. (1998). Cognitive and emotional differences between short and long-term vipassana meditators. Imagination, Cognition and Personality, 18(1), 69-81.

Ernst, E., Schmidt, K., \& Wider, B. (2005).CAM research in Britain: The last 10 years. Complementary Therapies in Clinical Practice, 11, 17-20

Esch, T., Fricchione, G. L., \& Stefano, G. B. (2003). The therapeutic use of the relaxation response in stress-related diseases. Medical Science Monitor, 9(2), RA23-34.

Galvin, J. A., Benson, H., Deckro, G. R., Fricchione, G. L., \& Dusek, J. A. (2006). The relaxation response: Reducing stress and improving cognition in healthy aging adults. Complementary Therapies in Clinical Practice, 12, 186-191.

Goleman, D. J. (1988). The meditative mind: The varieties of meditative experience. New York: G.P. Putnam's Sons.

Goleman, D. J. (2003). Como lidar com emoções destrutivas. Rio de Janeiro: Elsevier.
Goleman, D. J \& S Schwartz, G. E. (1976). Meditation as an intervention in stress reactivity. Journal of Consulting and Clinical Psychology, 44(3), 456-466.

Grossman, P., Niemannb, L., Schmidtc, S., \& Walachc, H. (2004). Mindfulness-based stress reduction and health benefits: A meta-analysis. Journal of Psychosomatic Research, 57, 35-43.

Guimarães, H. P., \& Avezum, A. (2007). O impacto da espiritualidade na saúde física. Revista de Psiquiatria Clínica, 34(Supl. 1), 88-94.

Hankey, A. (2006). Studies of advanced stages of meditation in the tibetan buddhist and vedic traditions. I: A comparison of general changes. Evidence-based Complementary and Alternative Medicine, 3(4), 513-521.

Hayward, J. W., \& Varela, F. J. (2001). Gentle bridges: Conversations with the Dalai Lama on the sciences of mind. Boston: Shambala.

Holmes, D. S. (1984). Meditation and somatic arousal reduction: A review of the experimental evidence. American Psychologist, 39(1), 1-10.

Infante, J. R., Peran, F., Martinez, M., Roldan, A., Poyatos, R., Ruiz, C. et al. (1998). ACTH and ß-endorphin in transcendental meditation. Physiology \& Behavior, 64(3), 311-315.

Jain, S., Shapiro, S. L., Swanick, S., Roesch, S. C., Mills, P. J., Bell, I., \& Schwartz, G. E. R. (2007). A randomized controlled trial of mindfulness meditation versus relaxation training: Effects on distress, positive states of mind, rumination and distraction. Annals of Behavioral Medicine, 33(1), 11-21.

Kabat-Zinn, J. (2003). Mindfulness-based interventions in context: Past, present, and future. Clinical Psychology: Science and Practice, 10(2), 144-156.

Kabat-Zinn, J., Wheeler, E., Light, T., Skillings, A., Scharf, M., \& Cropley, T. G. (1998). Influence of a mindfulness-based stress reduction intervention on rates of skin clearing in patients with moderate to severe psoriasis undergoing phototherapy (UVB) and photochemotherapy (PUVA). Psychosomatic Medicine, $60,625-632$

Kristeller, J. L., \& Hallett, B. (1999). An exploratory study of a meditation-based intervention for binge eating disorder. Journal of Health Psychology, 4(3), 357-363.

Lazar, S. W., Kerr, C. E., Wasserman, R. H., Gray, J. R., Greve, D. N., Treadway, M. T. et al. (2005). Meditation experience is associated with increased cortical thickness. Neuroreport, 16(17), 1893-1897.

Lehmann, D., Faber, P. L., Achermann, P., Jeanmonod, D., Gianotti, L. R. R., \& Pizzagalli, D. (2001). Brain sources of EEG gamma frequency during volitionally meditation-induced, altered states of consciousness, and experience of the self. Psychiatry Research: Neuroimaging Section, 108, 111-121.

Leung, Y., \& Singhal, A. (2004). An examination of the relationship between Qigong meditation and personality. Social Behavior and Personality, 32(4), 313-320.

Levine, M. (2000). The positive psychology of buddhism and yoga: Paths to a mature happiness. New Jersey: Lawrence Erlbaum Associates.

Lutz, A., Greischar, L. L., Rawlings, N. B., Ricard, M., \& Davidson, R. J. (2004). Long-term meditators self-induce high-amplitude gamma synchrony during mental practice. Proceedings of the National Academy of Sciences, 101(46), 16369-16373.

MacLean, C. R. K., Walton, K. G., Wenneberg, S. R., Levitsky, D. K., Mandarino, J. P., Waziri, R., et al. H. (1997). Effects of the transcendental meditation program on adaptive mechanisms: Changes in hormone levels and responses to 
stress after 4 months of practice. Psychoneuroendocrinology 22(4), 277-295.

Martin, J. R.(1997). Mindfulness: A proposed common factor. Journal of Psychotherapy Integration, 7(4), 291-312.

Maura, P., Donna, P., James, D. H., Ivan, V., Sanford, N., Maxwell, R. et al. (2006). Effects of a randomized controlled trial of transcendental meditation on components of the metabolic syndrome in subjects with coronary heart disease. Archives of Internal Medicine, 166(11), 1218-1224.

McCabe, P. (2005). Complementary and alternative medicine in Australia: A contemporary overview. Complementary Therapies in Clinical Practice, 11, 28-31.

Miller, J. J., Fletcher, K., \& Kabat-Zinn, J. (1995). Threeyear follow-up and clinical implications of a mindfulness meditation-based stress reduction intervention in the treatment of anxiety disorders. General Hospital Psychiatry, $17,192-200$

Ministério da Saúde. (2006). Portaria $n^{\circ}$ 971, de 3 de maio. Política Nacional de Práticas Integrativas e Complementares (PNPIC) no Sistema Único de Saúde, Departamento de Atenção Básica. Brasília, DF: Autor.

Nakao, M., Myers, P., Fricchione, G., Zuttermeister, P. C., Barsky, A. J., \& Benson, H. (2001). Somatization and symptom reduction through a behavioral medicine intervention in a mind/body medicine clinic. Behavioral Medicine, 26(4), 169-176.

Naranjo, C. (2005). Entre meditação e psicoterapia. Rio de Janeiro: Vozes

Newberg, A., Alavi, A., Baime, M., Pourdehnad, M., Santanna, J., \& D'Aquili, E. (2001). The measurement of regional cerebral blood flow during the complex cognitive task of meditation: A preliminary SPECT study. Psychiatry Research: Neuroimaging Section, 106, 113-122.

Oman, D., Hedberg, J. T., \& Thoresen, C. (2006). Passage meditation reduces perceveid stress in health professionals: $A$ randomized controlled trial. Journal of Consulting and Clinical Psychology, 74(4), 714-719.

Ostafin, B., Chawla, N., Bowen, S., Dillworth, T., Witkiewitz, K., \& Marlatt, G. (2006). Intensive mindfulness training and the reduction of psychological distress: A preliminary study. Cognitive and Behavioral Practice, 13, 191-197.

Peres, J. F. P., Simão, M. J. P., \& Nasello, A. G. (2007). Espiritualidade, religiosidade e psicoterapia. Revista de Psiquiatria Clínica, 34(Supl. 1), 136-145.

Schaffer, S. D., \& Yucha, C. B. (2004). Relaxation \& pain management: The relaxation response can play a role in managing chronic and acute pain. The American Journal of Nursing, 104(8), 75-82.

Schwartz, G. E., \& Davidson, R. J., \& Goleman, D. J. (1978). Patterning of cognitive and somatic processes in the selfregulation of anxiety: Effects of meditation versus exercise. Psychosomatic Medicine, 40(4), 321-329.

Shapiro, D. (1981). Meditation: Clinical and health-related applications. The Western Journal of Medicine, 134(2), 141-142.

Shapiro, S. L., Schwartz, G. E., \& Santerre, C. (2005). Meditation and positive psychology. In C. R. Snyder \& S. J. Lopez, Handbook of positive psychology (pp. 632-645). New York: Oxford USA Trade.

Slagter, H. A., Lutz, A., Greischar, L. L., Francis, A. D., Nieuwenhuis, S., Davis, J. M., \& Davidson, R. J. (2007).
Mental training affects distribution of limited brain resources. PLoS Biology, 5, 1-8.

Smith, J. C. (1975). Meditation as psychotherapy: A review of the literature. Psychological Bulletin, 82(4), 558-564.

Smith, J. C. (1978). Personality correlates of continuation and outcome in meditation and erect sitting control treatments. Journal of Consulting and Clinical Psychology, 46(2), 272-279.

Speca, M., Carlson, L. E., Goodey, E., \& Angen, M. (2000). A randomized, wait-list controlled clinical trial: The effect of a mindfulness meditation-based stress reduction program on mood and symptoms of stress in cancer outpatients. Psychosomatic Medicine, 62, 613-622.

Spiegel, D., Stroud, P., \& Fyfe, A. (1998). Complementary medicine. Western Journal of Medicine, 168, 241-247.

Sridevi, R., \& Krisha Rao, P. V. (1998). Temporal effects of meditation and personality. Psychological Studies, 43(3), 95-105.

Takahashi, T., Murata, T., Hamada, T., Omori, M., Kosaka, H., Kikushi, M. et al. (2005). Changes in EEG and autonomic nervous activity during meditation and their association with personality traits. International Journal of Psychophysiology, 55, 199-207.

Teasdale, J. D., Pope, M., Segal, Z. V., Moore, R. G., Hayhurst, H., \& Williams, S. (2002). Metacognitive awareness and prevention of relapse in depression: Empirical evidence. Journal of Consulting and Clinical Psychology, 70(2), 275-287.

Teasdale, J. D., Williams, J. M. G., Soulsby, J. M., Segal, Z. V., Ridgeway, V. A., \& Lau, M. A. (2000). Prevention of relapse/ recurrence in major depression by mindfulness-based cognitive therapy. Journal of Consulting and Clinical Psychology, 68(4), 615-623.

Travis, F., \& Wallace, R. K. (1999). Autonomic and EEG patterns during eyes-closed rest and transcendental meditation (TM) practice: The basis for a neural model of TM practice. Consciousness and Cognition, 8, 302-318.

Vandenbergue, L., \& Sousa, A. C. (2006). Mindfulness nas terapias cognitivas e comportamentais. Revista Brasileira de Terapias Cognitivas, 2(1), 35-44.

Wachholtz, A. B., \& Pargament, K. I. (2005). Is spirituality a critical ingredient of meditation? Comparing the effects of spiritual meditation, secular meditation, and relaxation on spiritual, psychological, cardiac, and pain outcomes. Journal of Behavioral Medicine, 28(4), 369-384.

Wallace, R. K. (1970). The physiological effects of transcendental meditation: A proposed fourth major state of consciousness. Scientific Research on the Transcendental Meditation Program: Collected Papers, 1, 43-78.

Wallace, R. K., \& Benson, H. The physiology of meditation. Scientific American, 226, 84-90.

Wallace, R. K., Benson, H., \& Wilson, A. F.(1971). A wakeful hypometabolic physiologic state. American Journal of Physiology, 221, 795-799. 